\title{
Hummingbird Sign and Squint Eyes
}

Ahmad Mursel Anam ${ }^{1}$, Raihan Rabbani², Farzana Shumy ${ }^{3}$

In mid-October, 2014, a 64-year-old lady presented with features of aspiration pneumonia. She has been being managed as a patient of "Parkinson-plus" syndrome for the past three years, including nasogastric tube feeding due to dysphagia. History from the carer revealed episodes of fall with postural instability, progressive rigidity of the body as well as dysphagia, and cognitive impairment, but no diplopia. Physical examination revealed a poor Mini Mental State Examination (MMSE) score, axial rigidity, and divergent squint (Image A). Chest findings, radiographs, and laboratory results were consistent with aspiration pneumonia, which was treated with antibiotics and supportive measures. But a diagnostic dilemma between Progressive Supranuclear Palsy (PSP) and late onset Chronic Progressive External Ophthalmoplegia (CPEO) with Parkinsonism remained for her progressive neurological condition. An MRI of brain was done, revealed typical "Hummingbird sign" (Image B) on mid-saggital section of T1-weighted image, as well as "squint eyes" sign (Image C) on axial section of T2-weighted image. So her diagnosis was established as PSP.

Due to symmetrical nature of ophthalmoplegia, diplopia may not be noticed by the patient in CPEO. Moreover, divergent squint is more common in CPEO, as horizontal gaze is affected more. On the other hand, vertical gaze is typically affected and horizontal gaze is spared, causing down-gaze palsy, in PSP. But, at the later stages, complete ophthalmoplegia may develop in PSP, giving rise to divergent squint, as well as "squint eyes" sign in the MRI. ${ }^{1}$

1. Dr. Ahmad Mursel Anam, MBBS

Chief Resident, ICU, Square Hospitals Ltd.

18/F, BU Qazi Nuruzzaman Sarak (West Panthapath), Dhaka 1205, Bangladesh.

2. Dr. Raihan Rabbani, MD (USA) Consultant, ICU, Square Hospitals Ltd.

18/F, BU Qazi Nuruzzaman Sarak (West Panthapath), Dhaka 1205, Bangladesh

3. Dr. Farzana Shumy, FCPS

Medical Officer, Dept. of Internal Medicine, Bangabandhu Sheikh Mujib Medical University Shahbagh, Dhaka 1000, Bangladesh.

\section{Corresponding author}

Dr. Ahmad Mursel Anam, MBBS

Chief Resident, ICU, Square Hospitals Ltd.

18/F, BU Qazi Nuruzzaman Sarak (West Panthapath), Dhaka 1205, Bangladesh.

Mailing address: House \# 61/B, Road \# 6/A, Dhanmondi RA, Dhaka 1209, Bangladesh

E-mail: murselanam@gmail.com

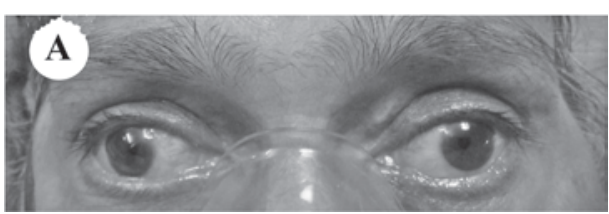

Image A: Divergent squint

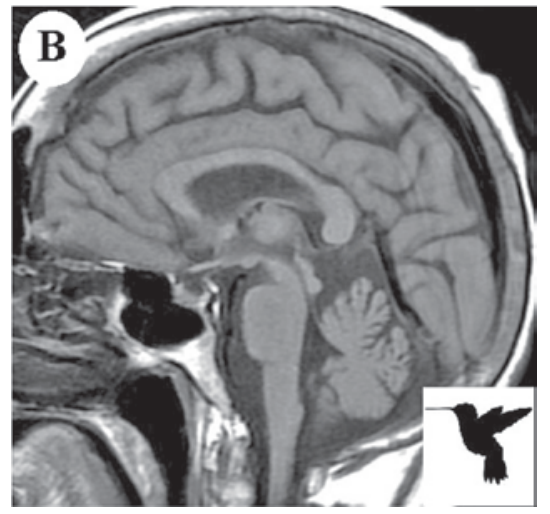

Image B: "Hummingbird sign": atrophy of the rostral midbrain tegmentum with relatively preserved pons in PSP, looks like the bill and body, respectively, of a hummingbird (inset) on a mid-saggital section of MRI brain.

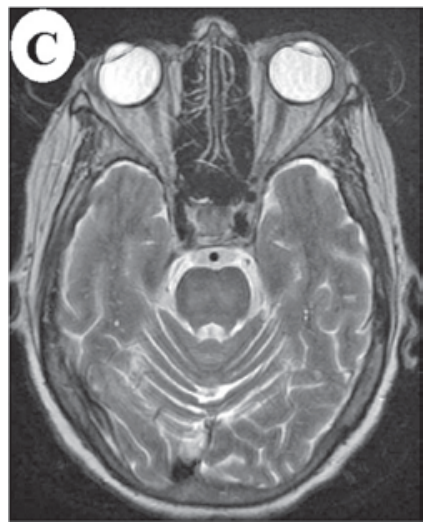

Image C: "Squint eyes" sign in the MRI.

In PSP, atrophy of the rostral midbrain tegmentum with relatively preserved pons, looks like the bill and body, respectively, of a hummingbird on a mid-saggital section of MRI brain, hence the term "Hummingbird sign". The "squint eyes" sign, signifies the ophthalmoplegia at the advanced stage of PSP. These two, among other MRI findings, are thought to be helpful for diagnosis of PSP. ${ }^{2}$

\section{References}

1. Barsottini OG, Felício AC, Aquino CC, Pedroso JL. Progressive Supranuclear Palsy: New Concepts. Arq Neuropsiquiatr 2010;68(6):938-946.

2. Abe K. Hummingbird and Squint Eyes in Progressive Supranuclear Palsy. Intern Med 2006;45(15):935-936. 